\title{
SUMS OF SQUARES OF LINEAR FORMS
}

\author{
José F. Fernando, Jesús M. Ruiz, and Claus Scheiderer
}

\begin{abstract}
Let $k$ be a real field. We show that every non-negative homogeneous quadratic polynomial $f\left(x_{1}, \ldots, x_{n}\right)$ with coefficients in the polynomial ring $k[t]$ is a sum of $2 n \cdot \tau(k)$ squares of linear forms, where $\tau(k)$ is the supremum of the levels of the finite non-real field extensions of $k$. From this result we deduce bounds for the Pythagoras numbers of affine curves over fields, and of excellent two-dimensional local henselian rings.
\end{abstract}

\section{Introduction}

Let $A$ be a commutative ring with unit. We consider quadratic forms with coefficients in $A$, that is, homogeneous polynomials of degree two

$$
f=f\left(x_{1}, \ldots, x_{n}\right)=\sum_{i, j=1}^{n} a_{i j} x_{i} x_{j}
$$

with $a_{i j} \in A$. If such $f$ is a sum of squares of linear forms, one may ask for the minimal number of squares needed to represent $f$. So we write

$$
\ell(f):=\inf \left\{r: \text { there are linear forms } h_{1}, \ldots, h_{r} \in A\left[x_{1}, \ldots, x_{n}\right] \text { with } f=\sum_{i=1}^{r} h_{i}^{2}\right\}
$$

(with the convention $\ell(f)=\infty$ if $f$ is not a sum of squares of linear forms), and put

$$
g_{n}(A):=\sup \left\{\ell(f): f \text { is a sum of squares of linear forms in } A\left[x_{1}, \ldots, x_{n}\right]\right\} .
$$

For $n=1, g_{1}(A)$ is nothing but the Pythagoras number of the ring $A$, usually denoted $p(A)$ : Every sum of squares of elements of $A$ is a sum of $p(A)$ squares, and $p(A)$ is the minimal number with this property $($ or $p(A)=\infty)$.

Our primary interest is in Pythagoras numbers for certain classes of rings. However, by an elementary observation originally due to Pfister, bounds for the $g_{n}$-invariants of a ring $A$ give bounds for the Pythagoras numbers of finite $A$-algebras (Lemma 2.3 below). This led us to the study of the invariants $g_{n}$.

Our main result concerns the case where $A=k[t]$, the polynomial ring in one variable over a real field $k$. Let

$$
\tau(k):=\sup \{s(E): E / k \text { finite, non-real }\},
$$

where $s(E)$ denotes the level of the field $E$, i.e. the smallest number $n$ such that -1 is a sum of $n$ squares in $E$. Since the level of a non-real field is a power of 2, according

Received by the editors April 22, 2006

2000 Mathematics Subject Classification. Primary 11E25; secondary 13J15, 14P99, 15A63.

Key words and phrases. Sums of squares, quadratic forms, level, Pythagoras numbers, local henselian rings. 
to Pfister's famous theorem, the invariant $\tau(k)$, if finite, is a power of 2 as well. It was first proved by Pfister that

$$
1+\tau(k) \leq p(k(t)) \leq 2 \tau(k)
$$

(see [L] p. 397). Cassels' theorem implies $p(k[t])=p(k(t))$, and so this gives $g_{1}(k[t])=$ $p(k[t]) \leq 2 \tau(k)$. We will prove that

$$
g_{n}(k[t]) \leq 2 n \cdot \tau(k)
$$

holds for all $n$ (Theorem 1.2). Also, we show that every positive semidefinite quadratic form over $k[t]$ is a sum of squares of linear forms. (See 1.1 for the notion of positive semidefiniteness.)

For $k=\mathbb{R}$ (or more generally, for $k$ a real closed field), the bound $g_{n}(k[t]) \leq 2 n$ had been proved around 1970 by Jakubović $[\mathrm{J}]$ and by Rosenblum and Rovnyak [RR] (note that $\tau(k)=1$ in this case). Different proofs were later given by Djoković [D] and by Choi, Lam and Reznick [CLR]. More recently, the first of us [F1] extended the result to the case where $k=$ Quot $\mathbb{R}\{x\}$, the quotient field of the ring of convergent power series in one variable (again, $\tau(k)=1$ in this case). While the proof in [F1] was inspired by Djoković's ideas from [D], our proof of the general case takes up the approach of [CLR].

Our main result, together with its proof, is presented in Section 1. Section 2 contains several applications. An immediate one is to Pythagoras numbers of affine curves (2.4). Somewhat less obvious is the application to the Pythagoras numbers of two-dimensional excellent local henselian rings. Indeed, assuming that the residue field $k$ of such a ring $A$ is real, we show $g_{n}(A) \leq 2 n \cdot \tau(k)$ if $A$ is regular (Proposition 2.7). Without any regularity assumption, this gives $p(A)<\infty$ whenever $\tau(k)<\infty$ (Theorem 2.9). We do not know whether conversely $p(A)<\infty$ implies $\tau(k)<\infty$, but at least we can show that $p(A)<\infty$ implies $\tau\left(k^{\prime}\right)<\infty$ for some finite real field extension $k^{\prime}$ of $k$ (2.11). The essential tool for the proofs of these results, apart from our main theorem, is the Artin approximation theorem. Finally, we discuss the lines between our results and the well-known weak and strong questions from [CDLR].

\section{The main result}

1.1. Let $A$ be a commutative ring with unit. See the introduction for basic notation. An element $a$ in $A$ is called positive semidefinite (or $p s d$, for short), if $\varphi(a) \geq 0$ holds for every homomorphism $\varphi$ from $A$ into an ordered field. In particular, this notion applies to polynomials over $A$. Using the Artin-Lang theorem from real algebra, one can rephrase the condition as follows: A polynomial $f$ in $A\left[x_{1}, \ldots, x_{n}\right]$ is psd if and only if, for every homomorphism $\varphi: A \rightarrow R$ into a real closed field $R$, the polynomial $f^{\varphi}$ over $R$ (obtained by applying $\varphi$ to the coefficients of $f$ ) takes non-negative values on $R^{n}$.

We will prove:

Theorem 1.2. Let $k$ be a real field. Then the bound

$$
g_{n}(k[t]) \leq 2 n \cdot \tau(k)
$$

holds for all $n$. Moreover, every psd quadratic form over $k[t]$ is a sum of squares of linear forms. 
Remark 1.3. For $k=\mathbb{R}$, the theorem says $g_{n}(\mathbb{R}[t]) \leq 2 n$. As mentioned before, this has been proved by several authors, and some of these approaches were even constructive ([D] and [CLR], see also [L] XIII.5). In [CDLR] (p. 50) the authors remark that the proof from [CLR] is valid as long as $k$ is a real closed field, but that it does not seem to generalize to other ground fields. In particular, they mention the case where $k$ is hereditarily pythagorean (this is equivalent to $\tau(k)=1$ ), and they are speculating that the bound $g_{n}(k[t]) \leq 2 n$ should remain valid in this case.

In $[\mathrm{F} 1]$, the bound $g_{n}(k[t]) \leq 2 n$ was proved for $k=$ Quot $\mathbb{R}\{x\}$. The approach of this paper can be adjusted to work for any hereditarily pythagorean field $k$, which settles the guess from [CDLR]. The basic idea in [F1] was to enlarge the polynomial ring $k[t]$ to $k(\sqrt{-1})[t]$, and to factor matrices over this larger ring, in order to produce the desired sums of squares decompositions. This was inspired by $[\mathrm{D}]$, and it used Djoković's theorem on dominant diagonalization.

It is possible to carry this method one step further: One can employ the polynomial ring $D[t]$ over $D=(-1,-1)_{k}$, the skew field of quaternions over $k$, and in this way prove the bound $g_{n}(k[t]) \leq 4 n$ for all fields $k$ satisfying $\tau(k) \leq 2$. Indeed, Djoković's construction for dominant diagonalization works for matrices over $D[t]$.

One might speculate to stretch the method even more, and use polynomials over octonions to arrive at a bound $g_{n}(k[t]) \leq 8 n$ for $\tau(k) \leq 4$. However, serious difficulties arise in this case caused by the lack of associativity, and it is not clear how to overcome them. At any rate, this case would definitely mean the end of this line, since it is well known that there are no composition algebras beyond dimension eight.

The proof given here works uniformly for any $k$, regardless of what $\tau(k)$ is, by reconsidering the approach from [CLR]. We point out that our proof is completely constructive as well, if one assumes that polynomials in $k[t]$ can be factored and that sums of squares decompositions of psd polynomials in $k[t]$ can be found.

1.4. For the proof of the theorem, we work with symmetric matrices instead of quadratic forms. Given a commutative ring $A$ containing $\frac{1}{2}$, we associate to any symmetric matrix $S \in \mathrm{M}_{n}(A)$ the quadratic form (homogeneous polynomial of degree two)

$$
F_{S}=F_{S}\left(x_{1}, \ldots, x_{n}\right)=x^{t} S x,
$$

where $x=\left(x_{1}, \ldots, x_{n}\right)^{t}$. In this way we identify quadratic forms in $n$ variables with symmetric $n \times n$-matrices. A sums of squares representation

$$
F_{S}=h_{1}^{2}+\cdots+h_{N}^{2}
$$

with linear forms $h_{1}, \ldots, h_{N} \in A\left[x_{1}, \ldots, x_{n}\right]$ corresponds to a product decomposition

$$
S=T^{t} \cdot T
$$

of the coefficient matrix $S$, with $T \in \mathrm{M}_{N \times n}(A)$ (the $i$-th row of $T$ consists of the coefficients of the linear form $h_{i}$ ). We will always write

$$
\ell(S):=\inf \left\{N: \text { there is } T \in \mathrm{M}_{N \times n}(A) \text { with } S=T^{t} T\right\}
$$

for the sums of squares length of the quadratic form $F_{S}$. Our proof makes use of some basic facts from quadratic form theory, such as Witt decomposition and the basic theory of Pfister forms. For all this we refer to $[\mathrm{L}]$. 
1.5. We now take up the proof of Theorem 1.2. So let $k$ be a real field. We always abbreviate $A:=k[t]$. For the proof we will assume that $\tau:=\tau(k)$ is finite, and will later show how the same arguments can be used for the qualitative part of the theorem disregarding the $\tau$-invariant. Here we know that every psd element of $A$ is a sum of $2 \tau$ squares of elements of $A$. Note that this fact corresponds precisely to the statement of the theorem in case $n=1$. We use it as the starting point for an induction on $n$.

Let $n>1$, and fix a psd symmetric matrix $S \in \mathrm{M}_{n}(A)$. Write $S$ as a block matrix

$$
S=\left(\begin{array}{cc}
a & u^{t} \\
u & S^{\prime}
\end{array}\right)
$$

with $a \in A, u \in A^{n-1}$ (vectors are columns) and a symmetric matrix $S^{\prime} \in \mathrm{M}_{n-1}(A)$. If $a=0$ then $u=0$, and we are done by induction. So assume $a \neq 0$. The polynomial $a \in A=k[t]$ is psd.

The discriminant of $F_{S}$ with respect to the variable $x_{1}$ is a quadratic form in $\left(x_{2}, \ldots, x_{n}\right)$, which corresponds to the symmetric matrix $D:=a S^{\prime}-u u^{t} \in \mathrm{M}_{n-1}(A)$. Since $S$ is psd, $D$ is psd as well. By the inductive hypothesis, $\ell(D) \leq 2(n-1) \tau$. Completing the square we see that the quadratic form $a F_{S}$ is a sum of $1+\ell(D) \leq 2 n \tau$ squares:

$$
a S=\left(\begin{array}{cc}
0 & 0^{t} \\
0 & D
\end{array}\right)+\left(\begin{array}{l}
a \\
u
\end{array}\right)\left(a u^{t}\right)
$$

Writing $m:=2 n \tau$ from now on, we have seen that there is an identity $a S=U^{t} U$ where $U \in \mathrm{M}_{m \times n}(A)$.

To prove the theorem, we have to find an identity $S=T^{t} T$ with $T \in \mathrm{M}_{m \times n}(A)$. This will be done by induction on the degree of $a$. If $a \in k^{*}$, then $a$ is a sum of $p(k) \leq$ $p(k(t)) \leq 2 \tau(k)=: 2^{r}$ squares in $k$. So the Pfister form $\varphi:=<1,1>^{\otimes r}$ represents $a$, hence $a \varphi \cong \varphi$ and $a(n \times \varphi) \cong n \times \varphi$. As $m=2^{r} n$, we conclude that there is $C \in \mathrm{GL}_{m}(k)$ with $a I=C C^{t}$. Consequently, $a S=U^{t} U$ implies $S=\left(C^{-1} U\right)^{t}\left(C^{-1} U\right)$. Let now $\operatorname{deg}(a) \geq 1$, and let $f$ be a monic irreducible polynomial dividing $a$. If the field $k[t] /(f)$ is real, then reduction of the identity $a S=U^{t} U$ modulo $(f)$ shows that the matrix $U$ is (coefficient-wise) divisible by $f$. Since $a$, being psd, is divisible by $f^{2}$, we may therefore cancel a factor $f^{2}$ in this case.

This leaves us with the case where the field $k[t] /(f)$ is nonreal. This is the main step of the proof. Note that $f$ is a sum of squares in this case, and more precisely, of $2 \tau$ squares of elements of $A$. We will show that there exists a product decomposition $U=B W$ with $B \in \mathrm{M}_{m}(A)$ and $W \in \mathrm{M}_{m \times n}(A)$, such that $B^{t} B=f I$. Once this is known we are done, since combining it with $a S=U^{t} U$ gives $a S=f W^{t} W$, and we can cancel $f$.

1.6. So let $f$ be a monic irreducible polynomial in $A=k[t]$, and let $U \in \mathrm{M}_{m \times n}(A)$ such that $U^{t} U$ is divisible by $f$. We identify matrices with linear maps, where matrices act on column vectors from the left. The submodule $\operatorname{im}(U)$ of $A^{m}$ is totally isotropic modulo $f$, in the sense that $f$ divides $\langle x, x\rangle=\sum_{j=1}^{m} x_{j}^{2}$ for every $x \in \operatorname{im}(U)$. Let $M$ be a submodule of $A^{m}$ which contains $\operatorname{im}(U)$ and which is maximal with respect to being totally isotropic modulo $f$. It suffices to find $B \in \mathrm{M}_{m}(A)$ with $\operatorname{im}(B)=M$ and $B^{t} B=f I$. Indeed, this will give a factorization $U=B W$ as desired. So it is enough to prove: 
Proposition 1.7. Let $k$ be a real field, let $f \in A=k[t]$ be a monic irreducible polynomial which is a sum of $2^{r}$ squares, and let $m$ be a multiple of $2^{r}$. For any submodule $M$ of $A^{m}$ which is totally isotropic modulo $f$ and which is maximal with respect to this property, there exists a matrix $B \in \mathrm{M}_{m}(A)$ with $\operatorname{im}(B)=M$ and $B^{t} B=f I$.

1.8. Let $\varphi$ denote the quadratic form $<1,1>^{\otimes r}$ over $k$ ("sum of $2^{r}$ squares"). Write $L:=k[t] /(f)$ in the following. Since the level of $L$ satisfies $s(L) \leq 2^{r-1}$, the quadratic form $\varphi_{L}$ over $L$ is hyperbolic. Since $m$ is a multiple of $2^{r}$, say $m=2^{r} n$, the form $m \times<1>_{L}=n \times \varphi_{L}$ is hyperbolic over $L$ as well. The reduction $\bar{M}$ of $M$ modulo $(f)$ is a maximal totally isotropic subspace of $L^{m}$ (with respect to the form $n \times \varphi_{L}$ ). Hence its dimension is $\operatorname{dim}_{L}(\bar{M})=\frac{m}{2}$.

We need the following easy lemma, whose proof will be supplied later.

Lemma 1.9. Let $A$ be a principal ideal domain, and let $\mathfrak{m} \neq(0)$ be a prime ideal of $A$. Let $M$ be a free $A$-module of finite rank, and let $V$ be a subspace of the $A / \mathfrak{m}$-vector space $\bar{M}=M / \mathfrak{m} M$. Then there exists a direct summand $P$ of $M$ with $\bar{P}=V$.

1.10. By this lemma we find a direct sum decomposition $A^{m}=P \oplus Q$ of $A$-modules with $\bar{M}=\bar{P}$. Since $M$ is maximal with respect to being totally isotropic modulo $f$, it follows that $M=P \oplus f Q$. From $\operatorname{dim}_{L}(\bar{M})=\frac{m}{2}$ we see $\operatorname{rk}(P)=\operatorname{rk}(Q)=\frac{m}{2}$.

Let $G \in \mathrm{M}_{m}(A)$ be the matrix which acts as the identity on $P$ and as multiplication by $f$ on $Q$. Then $\operatorname{det}(G)=f^{m / 2}$ and $\operatorname{im}(G)=M$. Since $M$ is totally isotropic modulo $f$, the product $G^{t} G$ is divisible by $f$, say $G^{t} G=f V$ with $V \in \mathrm{M}_{m}(A)$. Comparing determinants gives $\operatorname{det}(V)=1$, and in particular, $V \in \mathrm{GL}_{m}(A)$. On the other hand, $V$ is symmetric (and psd). Now we need to know that such $V$ can be diagonalized over $A$ :

Theorem 1.11 (Harder, Djoković). For any symmetric and invertible matrix $V \in$ $\mathrm{GL}_{m}(k[t])$, there exists an invertible matrix $Q \in \mathrm{GL}_{m}(k[t])$ such that $Q^{t} V Q$ has coefficients in $k$.

Proof. This result appears as Theorem 13.4.3 in Knebusch's paper [K] and is attributed to Harder. An elementary proof (by "Hermite's method") can be found in [S] (Thm. 6.3.3). A constructive proof is due to Djoković ([D] Prop. 4). His method provides an algorithm for diagonalizing $V$.

1.12. Let us come back to the situation in 1.10. From Theorem 1.11 we get $Q \in$ $\mathrm{GL}_{m}(A)$ such that $Q^{t} V Q=D$ lies in $\mathrm{GL}_{m}(k)$ (and is diagonal, if we want). The matrix $B:=G Q$ satisfies therefore $\operatorname{im}(B)=\operatorname{im}(G)=M$ and $B^{t} B=f D$.

Let $\psi$ denote the quadratic form over $k$ which is represented by $D$ (of dimension $\left.m=2^{r} n\right)$. By specializing at one point, we see that actually $\psi \cong n \times \varphi$. Indeed, substitute the value $t:=0$ in the identity $B^{t} B=f D$. Since $f(0) \neq 0$, we see $B(0) \in \mathrm{GL}_{m}(k)$, and so $f(0) \cdot \psi \cong n \times \varphi$ (over $k$ ). Since $\varphi$ is a Pfister form which represents $f(0)$, it follows that $\varphi \cong f(0) \cdot \varphi$, hence $\psi \cong n \times \varphi$, as we claimed.

This means that there exists a matrix $C \in \mathrm{GL}_{m}(k)$ with $D=C^{t} C$. Combining this with $B^{t} B=f D$ we get $f I=\tilde{B}^{t} \tilde{B}$, where $\tilde{B}=B C^{-1}$. Since $\operatorname{im}(\tilde{B})=M$, this finishes the proof of Theorem 1.2 in the case $\tau(k)<\infty$. See 1.14 below for the case where $\tau(k)=\infty$. 
1.13. Proof of Lemma 1.9. We may assume $M=A^{n}$ and $0<\operatorname{dim}(V)<n$. The proof is by induction on $\operatorname{dim}(V)$. First assume $\operatorname{dim}(V)=1$ (and $n \geq 2$ ). We are given $x=\left(x_{1}, \ldots, x_{n}\right) \in A^{n}$ with $x \notin \mathfrak{m} A^{n}$, and may assume $x_{1} \notin \mathfrak{m}$. Let $\mathfrak{p}_{1}, \ldots, \mathfrak{p}_{r}$ be those prime ideals of $A$ which contain $x_{1}$. Choosing $b \in \mathfrak{m}$ for which $x_{2}+b \notin \mathfrak{p}_{i}$ for $i=1, \ldots, r$, the vector $x^{\prime}=\left(x_{1}, x_{2}+b, \ldots\right)$ is unimodular.

For the inductive step let $V_{1} \subset V$ be a one-dimensional subspace, and let $P_{1}$ be a direct summand of $M$ such that $\bar{P}_{1}=V_{1}$. Choose a decomposition $M=P_{1} \oplus Q_{1}$, and choose a subspace $V_{2}$ of $\bar{Q}_{1}$ such that $V=V_{1} \oplus V_{2}$. By the inductive hypothesis there is a direct summand $P_{2}$ of $Q_{1}$ for which $V_{2}=\bar{P}_{2}$. So $P:=P_{1} \oplus P_{2}$ is a direct summand of $M$, and $\bar{P}=V$.

1.14. Proof of the qualitative part of Theorem 1.2. This asserts that every psd quadratic form over $A=k[t]$ is a sum of squares of linear forms. The arguments from the proof for $\tau(k)<\infty$ essentially carry over, as we briefly indicate:

The proof is again by induction on the number $n$ of variables. Given a psd quadratic form $F=F\left(x_{1}, \ldots, x_{n}\right)$ over $A$, we can assume as in 1.5 that there exists a psd polynomial $0 \neq a \in A$ for which $a F$ is a sum of squares. Choose $m$ to be a power of 2 which is so large that, on the one hand, $a F$ is a sum of $m$ squares, and on the other hand, the leading coefficient of $a$ as well as every monic irreducible psd polynomial dividing $a$ is a sum of $m$ squares. (It is well known that this last condition is equivalent to $a$ itself being a sum of $m$ squares, but we do not need this.) With this choice of the number $m$, the proof goes through as before, showing that $F$ itself is a sum of $m$ squares.

\section{Applications}

In this section, we assemble a series of examples, remarks and applications, in particular to Pythagoras numbers.

Example 2.1. If $A=k$ is a field, quadratic forms can be diagonalized, and this gives the bound $g_{n}(k) \leq n \cdot p(k)$ (which was originally observed by Pfister). For many fields, though, much better bounds are available [BLOP].

We mention here another easy case, the ring of formal power series $A=k \llbracket x \rrbracket$ over a real field $k$. As said above, one has $g_{n}(k((x))) \leq n \cdot p(k((x)))=n \cdot p(k)$. On the other hand, one gets immediately $g_{n}(k \llbracket x \rrbracket)=g_{n}(k((x)))$ by clearing denominators. (This is actually part of a more general statement for arbitrary real valuations, see Lemma 2.8.) Together this gives $g_{n}(k \llbracket x \rrbracket) \leq n \cdot p(k)$.

Remark 2.2. For fields of finite transcendence degree over their prime field or over a real closed field, upper bounds for $\tau(k)$ are available, although they may not always be sharp. For example, if $k$ is a real number field then $\tau(k)=4$ if there is a dyadic place $v$ for which $\left[k_{v}: \mathbb{Q}_{2}\right]$ is odd; otherwise, $\tau(k)=2$. (See $[\mathrm{L}]$, loc. cit.) If $k$ has transcendence degree $d$ over $\mathbb{Q}$ then $\tau(k) \leq 2^{d+2}$, using the Milnor conjecture as proved by Voevodsky. The better bound $\tau(k) \leq 2^{d+1}$ holds for $d \geq 2$ if Kato's cohomological Hasse principle is true. (See [Sch] 5.26 for a more detailed discussion.)

If $k$ has transcendence degree $d$ over a real closed field, then $\tau(k) \leq 2^{d}$ by a theorem of Pfister (see [L] XI.4.10).

Generally, the Milnor conjecture implies for any field $k$ that $\tau(k) \leq 2^{d}$, where $d=\operatorname{vcd}_{2}(k)=\operatorname{cd}_{2} k(\sqrt{-1})$ is the virtual cohomological 2-dimension of $k$. 
It is an open problem whether $p(k)<\infty$ implies $p(k(t))<\infty$ (or equivalently, $\tau(k)<\infty$ ) for every (real) field $k$.

Our applications of Theorem 1.2 are based on the following elementary lemma:

Lemma 2.3. Let $A$ be a ring, and let $B$ be an A-algebra which is generated by $n$ elements as an $A$-module. Then $p(B) \leq g_{n}(A)$.

The proof is straightforward, see [CDLR] $\S 2$. From this lemma and Theorem 1.2 we immediately get:

Theorem 2.4. Let $k$ be a real field with $\tau(k)<\infty$. Then $p(A)<\infty$ holds for every one-dimensional k-algebra $A$ of finite type.

Proof. By Noether normalization, $A$ is a finite $k[t]$-algebra.

Remarks 2.5. 1. For the record we note that if there is a homomorphism $k[t] \rightarrow A$ by which $A$ is generated by $n$ elements as a $k[t]$-module, then the bound $p(A) \leq 2 n \cdot \tau(k)$ holds.

2. A related kind of application of Theorem 1.2 is this: Let $f \in A\left[x_{1}, \ldots, x_{n}\right]$ be a form which is a sum of squares of forms of degree $d$. Then $f$ can be written as a sum of $g_{N}(A)$ such squares, where

$$
N=\left(\begin{array}{c}
n+d-1 \\
d
\end{array}\right)
$$

(see [CDLR] p. 51).

Now we turn to henselian local rings. To start with, we have:

Proposition 2.6. Let $A$ be an excellent henselian local ring. Then $g_{n}(A)=g_{n}(\widehat{A})$ holds for all $n$. In particular, $p(A)=p(\widehat{A})$.

Proof. The proof depends on the Artin approximation theorem, for which we refer to [KPP]. Suppose first that $g_{n}(\widehat{A})=\gamma$ is finite, and let $f \in A\left[x_{1}, \ldots, x_{n}\right]$ be a sum of squares of linear forms. Then we have

$$
f=\sum_{i=1}^{\gamma} g_{i}^{2}
$$

for some linear forms $g_{i} \in \widehat{A}\left[x_{1}, \ldots, x_{n}\right]$. Equating coefficients on both sides and looking at the coefficients of the $g_{i}$ as unknowns, we have a polynomial system of equations over $A$ that has a solution over $\widehat{A}$. Hence, by Artin approximation, it has a solution over $A$, and we find $h_{i} \in A\left[x_{1}, \ldots, x_{n}\right]$ such that $f=\sum_{i=1}^{\gamma} h_{i}^{2}$. We conclude that $g_{n}(A) \leq \gamma$.

Conversely, suppose $g_{n}(A)=\gamma<\infty$, and let $f \in \widehat{A}$ be a sum of squares of linear forms in $\widehat{A}$, say

$$
f=\sum_{j=1}^{r} g_{j}^{2}
$$

with linear forms $g_{j}$ over $\widehat{A}$. We try to find an identity

$$
f=\sum_{i=1}^{\gamma} h_{i}^{2}
$$


with linear forms $h_{i}$ over $\widehat{A}$. Again, this amounts to solving a polynomial system over $\widehat{A}$ whose unknowns are the coefficients of the $h_{i}$. By Artin approximation, it is enough to solve the system modulo $\widehat{\mathfrak{m}}^{k}$ for a suitable large $k \geq 1$ (here $\widehat{\mathfrak{m}}$ denotes the maximal ideal of $\widehat{A}$ ). Choose linear forms $\tilde{g}_{j}$ over $A$ such that all $\tilde{g}_{j}-g_{j}$ have coefficients in $\widehat{\mathfrak{m}}^{k}$. Then $\tilde{f}:=\sum_{i=1}^{r} \tilde{g}_{j}^{2}$ is a sum of squares of linear forms in $A\left[x_{1}, \ldots, x_{n}\right]$, and consequently, a sum of $\gamma$ squares, say

$$
\tilde{f}=\sum_{i=1}^{\gamma} \tilde{h}_{i}^{2}
$$

with suitable linear forms $\tilde{h}_{i}$ over $A$. Since $\tilde{f}-f$ has coefficients in $\widehat{\mathfrak{m}}^{k}$, this solves (*) modulo $\widehat{\mathfrak{m}}^{k}$, as desired. We are done.

Proposition 2.7. Let $A$ be an excellent henselian local ring. Suppose that $A$ is regular of dimension two and has a real residue field $k$. Then $g_{n}(A) \leq 2 n \cdot \tau(k)$ for all $n$.

The proof uses the following simple observation:

Lemma 2.8. Let $B$ be a valuation ring with real residue field and with quotient field $F$. Then $g_{n}(B[y])=g_{n}(F[y])$.

Proof. It suffices to see that if $B[y]\left[x_{1}, \ldots, x_{n}\right]$ contains a sum of squares of linear forms from $F[y]\left[x_{1}, \ldots, x_{n}\right]$, then $B[y]\left[x_{1}, \ldots, x_{n}\right]$ contains the linear forms themselves. Looking at the coefficients of the forms involved, one sees that it suffices to show that if $f=\sum_{i} f_{i}^{2} \in B[y]$, with $f_{i} \in F[y]$, then each $f_{i}$ lies in fact in $B[y]$. To show this, let $f_{i}=\sum_{j} a_{i j} y^{j} \in F[y]$, and let $a_{\lambda \mu}$ be a coefficient with smallest valuation. Suppose by way of contradiction that $a=1 / a_{\lambda \mu} \in \mathfrak{m}$, where $\mathfrak{m}$ is the maximal ideal of $B$. Then $a f_{i} \in B[y]$ for all $i$, and $a f_{\lambda} \notin \mathfrak{m}[y]$. Since $\mathfrak{m}[y]$ is a real prime ideal of $B[y]$, we conclude that $a^{2} f=\sum_{i}\left(a f_{i}\right)^{2} \notin \mathfrak{m}[y]$. Consequently, $a \notin \mathfrak{m}$, which is the desired contradiction.

Proof of Proposition 2.7. We can assume $\tau(k)<\infty$. By Proposition 2.6 we can suppose $A$ is complete, hence $A=k \llbracket x, y \rrbracket$. Since clearly $\tau(k((x)))=\tau(k)$ ([Sch] 5.13), our main theorem gives $g_{n}(k((x))[y]) \leq 2 n \cdot \tau(k)$ for all $n$. From the lemma we get $g_{n}(k \llbracket x \rrbracket[y]) \leq 2 n \cdot \tau(k)$. We end with another application of Proposition 2.6, since $k \llbracket x, y \rrbracket$ is the completion of $k \llbracket x \rrbracket[y]_{(x, y)}$.

Finally we prove:

Theorem 2.9. Let $A$ be any excellent henselian local ring of dimension two whose residue field $k$ satisfies $\tau(k)<\infty$. Then the Pythagoras number $p(A)$ is finite.

Proof. If $k$ is nonreal then -1 is a sum of squares in $A$, and the assertion is clear. So we assume that $k$ is real. Let $\widehat{A}$ be the completion of $A$. This is a complete noetherian local ring with $\operatorname{dim}(\widehat{A})=2$. Such a ring is known to be a finite $k \llbracket x, y \rrbracket$-algebra, essentially by the Cohen structure theorem (see [Bo] ch. IX $\S 3$ Théorème 2 ). From Proposition 2.7 (and Lemma 2.3) we therefore get $p(\widehat{A})<\infty$, and thus $p(A)<\infty$ by Proposition 2.6. 
Remark 2.10. With this last result, the understanding of the Pythagoras numbers of excellent local henselian rings has become quite precise. To describe the situation, denote by $\operatorname{dim}_{r}(A)$ the real dimension of $A$ (see [FRS]). For $A$ an excellent local henselian $\operatorname{ring}, \operatorname{dim}_{r}(A)=\max \{\operatorname{dim}(A / \mathfrak{p}): \mathfrak{p}$ is a real prime ideal of $A\}$. Clearly $\operatorname{dim}_{r}(A) \leq \operatorname{dim}(A)$, but equality need not hold in general. By the main result of [FRS], $p(A)=\infty$ if $\operatorname{dim}_{r}(A) \geq 3$. On the other hand, for studying $p(A)$ one can replace $A$ by a quotient $A / I$ with $\operatorname{dim}(A / I)=\operatorname{dim}_{r}(A)$, in such a way that bounds for $p(A / I)$ provide bounds for $p(A)$ (this goes back to [CDLR], see also [F2] p. 1910). Thus we are left with the case where $\operatorname{dim}(A)=\operatorname{dim}_{r}(A)$ is 1 or 2 .

The one-dimensional case can be considered folklore: After completion, $A$ is a finite module over some power series ring, and the second part of Remark 2.1 applies to bound $p(A)$ in terms of $p(k)$. Consequently, $p(A)$ is finite iff $p(k)$ is so. In the same vein, for $\operatorname{dim}(A)=2$, Theorem 2.9 shows that $p(A)$ is bounded in terms of $\tau(k)$. We deduce that $p(A)$ is finite if $\tau(k)$ is so. Without going into details we point out that these bounds can be made explicit, involving $p(k)$ or $\tau(k)$ and suitable multiplicities of $A$ (see [F1]).

Conversely, if $p(A)$ is finite then $p(k)$ is also finite by trivial reasons. Whether or not $\tau(k)$ is finite is not clear. Here we touch the well-known open problem of deciding whether $p(k)<\infty$ implies $p(k(t))<\infty$. The closest we can come to is this:

Proposition 2.11. Let $A$ be local henselian and excellent, with real residue field $k$. If $\operatorname{dim}_{r}(A)=2$ and $p(A)<\infty$, there exists a finite real extension $k^{\prime}$ of $k$ such that $\tau\left(k^{\prime}\right)<\infty$.

Proof. We may assume that $A$ is a real domain of Krull dimension 2. Then there is a sequence of quadratic transforms $A \rightarrow A^{\prime}$ in the field of fractions $K$ of $A$, such that $A^{\prime}$ is regular of dimension 2 and its residue field $k^{\prime}$ is a real finite extension of $k$ [AR]. Clearly, $p(A)<\infty$ implies $p(K)<\infty$. By [Sch] 5.16(b), applied to $A^{\prime}$, we get $p\left(k^{\prime}(t)\right) \leq p(K)$, and so $\tau\left(k^{\prime}\right)<\infty$.

Remark 2.12. Although it is quite obvious, we would like to briefly sketch the line between our results and questions $\left(Q_{1}\right)$ and $\left(Q_{2}\right)$ from [CDLR] (p. 49), commonly referred to as "weak question" and "strong question". Given a ring $A$ with $p(A)<\infty$ and a finite $A$-algebra $B$, the weak question asks whether $p(B)$ is necessarily finite. The strong question asks whether even $p(B) \leq n \cdot p(A)$ holds, if $B$ is generated by $n$ elements as an $A$-module. To our knowledge, no single counterexample is known to date, not even to the strong question.

It is clear that the weak question has a positive answer whenever $g_{n}(A)$ is finite for all $n$. Thus, our results provide such a positive answer for important new classes of rings $A$, in particular finitely generated $k$-algebras of transcendence degree one and local excellent henselian rings of dimension $\leq 2$ with residue field $k$, both in the case where the field $k$ satisfies $\tau(k)<\infty$. For the same rings, although in general our results do not suffice to imply a positive answer to the strong question in its original form, they imply that this question states the correct order of magnitude for $p(B)$, namely up to a constant factor independent of $n$. For example, if $A=k[t]$ is the polynomial ring over a real field, with $p(A)<\infty$, then $\tau(k)<2 p(A)$, and hence $p(B)<4 n \cdot p(A)$ whenever $B$ is generated by $n$ elements as an $A$-module. 
Note added in proof: In a recent preprint, D. Leep proves that $g_{n}(k[t])=g_{n}(k(t))$ holds. Using this, one can improve the bound $g_{n}(k[t]) \leq 2 n \cdot \tau(k)$ of Theorem 1.2 for many concrete fields $k$.

\section{References}

[AR] C. Andradas and J.M. Ruiz, On local uniformization of orderings, Contemp. Math. 155 (1994) 19-46.

[BLOP] R. Baeza, D. Leep, M. O'Ryan, and J. P. Prieto, Sums of squares of linear forms, Math. Z. 193 (1986) 297-306.

[Bo] N. Bourbaki, Algèbre Commutative, Chapitres 8 et 9. Masson, Paris, 1983.

[CDLR] M.D. Choi, Z.D. Dai, T.Y. Lam, and B. Reznick, The Pythagoras number of some affine algebras and local algebras, J. Reine Angew. Math. 336 (1982) 45-82.

[CLR] M.D. Choi, T.Y. Lam, and B. Reznick, Real zeros of positive semidefinite forms. I, Math. Z. 171 (1980) 1-26.

[D] D.Ž. Djoković, Hermitian matrices over polynomial rings, J. Algebra 43 (1976) 359-374.

[F1] J.F. Fernando, On the Pythagoras numbers of real analytic rings, J. Algebra 243 (2001) 321-338.

[F2] _ Sums of squares in real analytic rings, Trans. Am. Math. Soc. 354 (2002) 19091919.

[FRS] J.F. Fernando, J.M. Ruiz, and C. Scheiderer, Sums of squares in real rings, Trans. Am. Math. Soc. 356 (2004) 2663-2684.

[J] V.A. Jakubović, Factorization of symmetric matrix polynomials (Russian), Dokl. Akad. Nauk SSSR 194 (1970) 532-535.

[K] M. Knebusch, Grothendieck- und Wittringe von nichtausgearteten symmetrischen Bilinearformen, S.-B. Heidelberger Akad. Wiss. Math.-Natur. Kl. 1969/70 93-157.

[KPP] H. Kurke, G. Pfister, D. Popescu, M. Roczen, and T. Mostowski, Die Approximationseigenschaft lokaler Ringe, Lecture Notes in Mathematics, Vol. 634. Springer-Verlag, Berlin-New York, 1978.

[L] T.Y. Lam, Introduction to quadratic forms over fields, Graduate Studies in Mathematics, 67. American Mathematical Society, Providence, RI, 2005.

[RR] M. Rosenblum and J. Rovnyak, The factorization problem for nonnegative operator valued functions, Bull. Am. Math. Soc. 77 (1971) 287-318.

[S] W. Scharlau, Quadratic and Hermitian forms. Grundlehren der mathematischen Wissenschaften 270, Springer, Berlin, 1985.

[Sch] C. Scheiderer, On sums of squares in local rings. J. Reine Angew. Math. 540 (2001) 205227.

Department of Mathematics, Autonomous University of Madrid, 28049 Madrid, Spain

E-mail address: josefrancisco.fernando@uam.es

Department of Geometry and Topology, Universidad Complutense de Madrid, 28040 MADRID, SPAIN

E-mail address: jesusr@mat.ucm.es

FB Mathematik und Statistik, Universität Konstanz, D-78457 Konstanz, Germany

E-mail address: claus.scheiderer@uni-konstanz.de 